\title{
Studying the leptonic structure of galaxy cluster atmospheres from the spectral properties of the SZ effect
}

\author{
S. Colafrancesco ${ }^{1,2,3}$, D. Prokhorov ${ }^{4,5}$, and V. Dogiel ${ }^{6}$ \\ 1 ASI Science Data Center, ASDC c/o ESRIN, via G. Galilei, 00044 Frascati, Italy \\ 2 ASI, Viale Liegi 26, Roma, Italy \\ 3 INAF - Osservatorio Astronomico di Roma, via Frascati 33, 00040 Monteporzio, Italy \\ e-mail: cola@mporzio.astro.it \\ 4 Moscow Institute of Physics and Technology, Institutskii lane, 141700 Moscow Region, Dolgoprudnii, Russia \\ 5 Institut d'Astrophysique de Paris, CNRS, UMR 7095, Université Pierre et Marie Curie, 98bis Bd Arago, 75014 Paris, France \\ ${ }^{6}$ Lebedev Physical Institute, 117924 Moscow, Russia
}

Received 2 May 2008 / Accepted 21 October 2008

ABSTRACT

\begin{abstract}
We study the energetics of galaxy-cluster atmospheres by analyzing the SZ effect spectra around the crossover frequency. We calculate analytically the expressions of both the crossover frequency and the spectral slope of the SZE around the crossover frequency in various cases: a thermal electron population; a power-law, non-thermal, electron population; and a population of electrons experiencing a stochastic acceleration. We find that the value of the crossover frequency $X_{0}$ of the SZE depends significantly on the cluster peculiar velocity $V_{r}$ which determines the amplitude of the kinematic SZE), while the value of the slope of the SZE does not depend on the kinematic SZE spectrum in the optimal frequency range around the crossover frequency of the thermal SZE, i.e. in the frequency range $x=3.5-4.5$. Therefore, while the amplitude of the $\mathrm{SZ}_{\mathrm{kin}}$ produces a systematic bias in the position of the crossover frequency $X_{0}$, it does not affect significantly the spectral slope of the SZE. We therefore propose using measurements of the spectral slope of the SZE to obtain unbiased information about the specific properties of various electron distributions in galaxy clusters as well as in other cosmic structures in which a SZE can be produced.
\end{abstract}

Key words. cosmology: theory - galaxies: clusters: general - cosmology: cosmic microwave background

\section{Introduction}

The SZ effect (hereafter SZE) is a promising tool for studying the complex physics of cluster atmospheres because it is sensitive to the specific features of the spectra of the various electron populations responsible for the CMB photon Comptonization (see e.g. Colafrancesco 2007, for a review). A SZE can be produced by different types of leptonic plasmas in astrophysical environments, such as: thermal (hot and/or warm) electrons in the atmospheres of galaxies and galaxy clusters (Birkinshaw 1999; Itoh et al. 1998; Colafrancesco et al. 2003); non-thermal (and relativistic) electrons in clusters and in the cavities produced by AGN radio lobes (Ensslin \& Kaiser 2000; Colafrancesco et al. 2003; Colafrancesco 2005); secondary electrons produced by Dark Matter annihilation in cosmic structures (Colafrancesco 2004), in addition to the kinematic SZE related to the bulk motion of these plasmas.

For the majority of galaxy clusters the most prominent form of SZE is the thermal one, which provides a CMB intensity change of

$\Delta I_{\mathrm{th}}=\frac{2\left(k T_{0}\right)^{3}}{(h c)^{2}} y_{\mathrm{th}} g(x)$

as produced by inverse Compton scattering (ICS) of $\mathrm{CMB}$ photons off the thermal electron population residing in the cluster atmosphere with a Comptonization parameter $y_{\text {th }}=$ $\left(\sigma_{\mathrm{T}} / m_{\mathrm{e}} c^{2}\right) \int \mathrm{d} \ell n_{\mathrm{e}} k_{\mathrm{B}} T_{\mathrm{e}}$ given in terms of the electron plasma number density $n_{\mathrm{e}}$ and temperature $T_{\mathrm{e}}$ (see e.g. Birkinshaw 1999, for a review and Colafrancesco et al. 2003, for a general derivation) and in terms of fundamental constants, namely the Thomson cross-section $\sigma_{\mathrm{T}}$, the electron mass $m_{\mathrm{e}}$, the speed of light $c$, the Boltzmann constant $k_{\mathrm{B}}$ and the Planck constant $h$. The function $g(x)$ depends on the adimensional frequency $x \equiv h v / k_{\mathrm{B}} T_{0}$, where $T_{0}=2.726 \mathrm{~K}$ is the CMB temperature, containing all the spectral information about the SZE (see e.g. Colafrancesco et al. 2003, for details).

There are three basic spectral features that characterize the thermal SZE signal:

i) a minimum in its intensity located at a frequency

$$
\begin{aligned}
x_{\mathrm{th}, \min } \approx & 2.265\left(1-0.0927 \theta_{\mathrm{e}}+2.38 \theta_{\mathrm{e}}^{2}\right) \\
& +\tau\left(-0.00674+0.466 \theta_{\mathrm{e}}\right),
\end{aligned}
$$

where $\theta_{\mathrm{e}} \equiv k T_{\mathrm{e}} / m_{\mathrm{e}} c^{2}$, and $\tau=\sigma_{\mathrm{T}} \int \mathrm{d} \ell n_{\mathrm{e}}$ is the optical depth of the electron plasma, whose exact position depends weakly on the electron spectrum (i.e. on the electron temperature $T_{\mathrm{e}}$ and number density $n_{\mathrm{e}}$ ) and equals $\sim 2.26$;

ii) a crossover frequency, $X_{0}$, whose value depends on the electron pressure/energy density and the electron optical depth

$X_{\mathrm{th}, 0} \approx a\left(T_{\mathrm{e}}\right)+\tau b\left(T_{\mathrm{e}}\right)$

with $a\left(T_{\mathrm{e}}\right)=3.830\left(1+1.162 \theta_{\mathrm{e}}-0.8144 \theta_{\mathrm{e}}^{2}\right)$ and $b\left(T_{\mathrm{e}}\right)=$ $3.021 \theta_{\mathrm{e}}-8.672 \theta_{\mathrm{e}}^{2}$, and is found at a frequency $>3.83$ for increasing values of $T_{\mathrm{e}}$ (the value $X_{\mathrm{th}, 0}=3.83$ is found in the non-relativistic limit, or in the limit $T_{\mathrm{e}} \rightarrow 0$ ); 
iii) a maximum of its intensity whose frequency location is

$$
\begin{aligned}
& x_{\text {th, } \max } \approx 6.511\left(1+2.41 \theta_{\mathrm{e}}-4.96 \theta_{\mathrm{e}}^{2}\right) \\
&+\tau\left(0.0161+8.16 \theta_{\mathrm{e}}-35.9 \theta_{\mathrm{e}}^{2}\right)
\end{aligned}
$$

and depends sensitively on the nature of the electron population and on its energy (momentum) spectrum (see Dolgov et al. 2001, for the case of electrons with a thermal spectrum; see also Colafrancesco et al. 2003, and Colafrancesco 2004, 2005, 2007, for the case of electron populations with different spectra).

Since the frequency location of the zero in every SZE signal contains a crucial dependence on the pressure (or energy density) of the electron population (see e.g. Eq. (3) for the thermal case; see also Colafrancesco et al. 2003, for the discussion of the general cases), it has been widely proposed to use this property to measure e.g. the cluster temperature (for a thermal electron population) or the pressure/energy density in the case of a more general electron population.

However, observations of the SZE close to the crossover frequency are biased due to the presence of the kinematic SZE

$\Delta I_{\text {kin }}=-\frac{2\left(k T_{0}\right)^{3}}{(h c)^{2}} \tau \frac{V_{r}}{c} h(x)$

(see Sunyaev \& Zel'dovich 1980; see also Eq. (8) below), which is associated with the peculiar velocity $V_{r}$ of the cluster. The unknown value of $V_{r}$ therefore limits the ability to measure the cluster temperature (or more generally its energy density) directly through the displacement of the crossover frequency $X_{0}$ of the SZE in the correct relativistic treatment. The possible presence of multiple sources of SZE in galaxy clusters (e.g. the thermal SZE, the SZE due to an additional warm gas component, the non-thermal SZE due to a relativistic plasma, the DM-induced SZE; see Colafrancesco 2007, for a recent review) could provide further uncertainties and biases in the measurement of the crossover frequency $X_{0}$ of the dominant thermal SZE, since they add to the thermal SZE with specific spectral shapes and amplitudes of their signals.

This parameter degeneracy might be broken - in principle - by observing the SZE signal at many frequencies, a procedure that is limited, however, by the observational setup of the current SZ experiments operating in a limited number (usually $~ 3-4$ ) of frequency bands of relatively wide frequency width $(\sim 15-30 \%$ of the band central frequency) requiring precise inter-calibration.

In this paper we explore the possibility of extracting unbiased information about the nature of the electron population that produces the SZE from the spectral shape of the relative SZE spectrum.

One of the frequency region where large variation of the SZE spectra are found is around the crossover frequency of the thermal SZE, at frequency $220 \mathrm{GHz}$ (see Fig. 1 in Colafrancesco 2007). Therefore, this is a favorable region for performing a spectral analysis of various SZE components with the aim to separate the contributions of various electron populations.

To provide quantitative estimates of our proposal that could be tested by SZE experiments with spectroscopic capabilities, we will present an analytical derivation of the spectral slope of various sources of SZE around the crossover frequency of the thermal SZE, i.e. in the range $x=3.5-4.5$ (corresponding to the frequency range $\sim 200-255 \mathrm{GHz}$ ). We show that, in this frequency region, the SZE spectral slopes are mostly independent of the cluster peculiar velocity, at variance with the value of the crossover frequency $X_{0}$, that is inevitably biased by the unknown

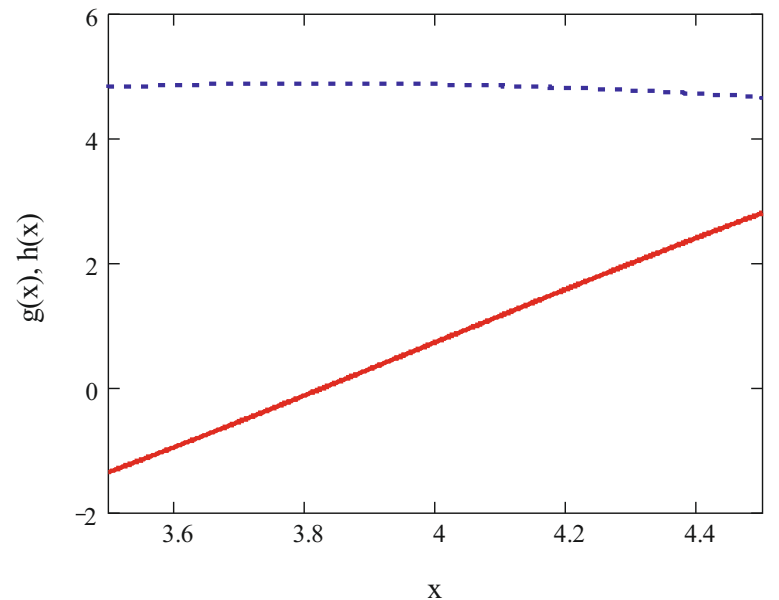

Fig. 1. The functions $g_{\mathrm{nr}}(x)$ (solid) and $h_{\mathrm{nr}}(x)$ (dashed) are shown in the frequency range $x=3.5-4.5$.

value of the cluster peculiar velocity $V_{r}$. For the sake of illustration, we first discuss in Sect. 2 the cases of the non-relativistic and relativistic formulations of the thermal SZ effect. In Sect. 3 we discuss the case of a completely non-thermal, relativistic SZE for a power-law electron spectrum. In Sect. 4 we discuss the physical case of the SZE produced by a Maxwellian electron distribution experiencing a stochastic acceleration process. We discuss our results and draw our conclusions in Sect. 5.

Throughout the paper, we use a flat, vacuum-dominated, CDM cosmology with $H_{0}=70 \mathrm{~km} \mathrm{~s}^{-1} \mathrm{Mpc}^{-1}, \Omega_{\mathrm{M}}=0.3$, $\Omega_{\Lambda}=0.7$.

\section{The spectrum of the thermal SZE around the crossover frequency}

The spectra of the thermal SZE in the relativistic and nonrelativistic limits differ quite significantly around the crossover frequency for increasing values of thermal plasma temperature (see e.g. Birkinshaw 1999, and references therein). This fact has been widely indicated as a possible means of extracting physical information, such as e.g. the electronic plasma temperature $T_{\mathrm{e}}$ from a measurement of the crossover frequency $X_{0}\left(T_{\mathrm{e}}\right)$ of the SZE spectrum, if the cluster peculiar velocity $V_{r}$ is known to a sufficiently high accuracy. However, cluster peculiar velocity information is often uncertain or even missing, which means that this technique can be affected by strong systematic biases.

In the following, we show that measuring the spectral slope of the SZE spectrum around the crossover frequency provides a means of extracting information on the electron distribution that is independent of the cluster peculiar velocity.

We compare the spectral slopes of the thermal SZE, SZ $\mathrm{th}_{\mathrm{th}}$, and the kinematic SZE, $\mathrm{SZ}_{\mathrm{kin}}$, calculated for an isothermal cluster in the frequency range $200-255 \mathrm{GHz}$, corresponding to the range $x \approx 3.5-4.5$ of the adimensional frequency $x \equiv h v / k T_{0}$.

We begin, for the sake of clarity, our discussion from the non-relativistic case where the Kompaneets (1957) equation can be solved analytically and leads, following Eq. (1), to an analytic expression for the $\mathrm{CMB}$ intensity change in the non-relativistic regime

$\Delta I_{\mathrm{th}, \mathrm{nr}}=\frac{2\left(k T_{0}\right)^{3}}{(h c)^{2}} \tau \frac{k T_{\mathrm{e}}}{m_{\mathrm{e}} c^{2}} g_{\mathrm{nr}}(x)$

with

$g_{\mathrm{nr}}(x)=\frac{x^{4} \mathrm{e}^{x}}{\left(\mathrm{e}^{x}-1\right)^{2}}\left(x \cdot \frac{\mathrm{e}^{x}+1}{\mathrm{e}^{x}-1}-4\right)$ 
The subscript " $n r$ " denotes the fact that the previous expression was obtained in the non-relativistic limit.

The kinematic SZE intensity change (Sunyaev \& Zeldovich 1980), due to the bulk motion of the cluster gas with peculiar velocity $V_{r}$ along the los, writes as

$\Delta I_{k}=-2 \frac{\left(k T_{0}\right)^{3}}{(h c)^{2}} \tau \frac{V_{r}}{c} h(x)$

with

$h_{\mathrm{nr}}(x)=\frac{x^{4} \mathrm{e}^{x}}{\left(\mathrm{e}^{x}-1\right)^{2}}$.

In the following, we use the quantity $i(x)$ to represent the adimensional CMB intensity spectral distortion

$i_{\mathrm{th}, k}(x)=\frac{\Delta I_{\mathrm{th}, k}}{\left[\frac{2\left(k T_{0}\right)^{3}}{(h c)^{2}} \tau\right]}$,

which contains relevant spectral information on the thermal and kinematic SZE.

The total SZE intensity in the case of a cluster with a single thermal electron population moving along the line of sight with a peculiar velocity $V_{r}$ is due to the combination of $\mathrm{SZ}_{\text {th }}$ and $\mathrm{SZ}_{\text {kin }}$ and is given by

$i(x)=\frac{k T_{\mathrm{e}}}{m_{\mathrm{e}} c^{2}}\left[g(x)-\frac{V_{r}}{c} \frac{m_{\mathrm{e}} c^{2}}{k T_{\mathrm{e}}} h(x)\right]$.

The series expansions of $g(x)$ and $h(x)$ around the adimensional frequency $x=4$ (see Appendix A) provides the values of the spectral slope $S$ and of the crossover frequency $X_{0}$.

The slope of the SZE spectrum around the crossover frequency $X_{0}$ is defined by the quantity

$S=\frac{i(x)-i\left(X_{0}\right)}{x-X_{0}}=\frac{i(x)}{x-X_{0}}$.

In Fig. 1, we compare the functions $g(x)$ and $h(x)$ in the frequency range of our interest. Since the $\mathrm{SZ}_{\text {kin }}$ spectral shape $h(x)$ is almost flat in the frequency range $x \approx 3.5-4.5$, the slope $S$ of the thermal SZE can be used to extract unbiased information about the nature of the electron population, i.e., in this case, its temperature $T_{\mathrm{e}}$.

In the non-relativistic limit, we find (see the Appendix for a derivation) that the slope of the total SZE (thermal plus kinematic) is given by

$S_{\mathrm{nr}}=\frac{k T_{\mathrm{e}}}{m_{\mathrm{e}} c^{2}} \frac{g\left(X_{0}+\delta x\right)}{\delta x} \approx 4.25 \frac{k T_{\mathrm{e}}}{m_{\mathrm{e}} c^{2}}=0.08 \frac{k T_{\mathrm{e}}}{10 \mathrm{keV}}$

and does not depend on the cluster peculiar velocity but only on the electron temperature $T_{\mathrm{e}}$.

The crossover frequency of the total SZE

$X_{0, \text { total, nr }}=3.83+0.193\left(\frac{V_{r}}{10^{3} \mathrm{~km} \mathrm{~s}^{-1}} \cdot \frac{10 \mathrm{keV}}{k T_{\mathrm{e}}}\right)$,

obtained from the condition $i\left(X_{0}\right)=0$ (see Appendix A.1) depends, instead, on both $k T_{\mathrm{e}}$ and $V_{r}$, so that a measure of the crossover frequency for the thermal SZE cannot provide an unambiguous estimate of the cluster temperature $T_{\mathrm{e}}$. Specifically, for values $k T_{\mathrm{e}}=10 \mathrm{keV}$ and $V_{r}= \pm 1000 \mathrm{~km} \mathrm{~s}^{-1}$, the values of $X_{0, \text { total,nr }}$ is found to be in the range 3.64-4.02, while for $V_{r}=0$ one recovers the non-relativistic value $X_{0, \text { total,nr }}=3.83$, which, obviously, does not depend on the cluster temperature.

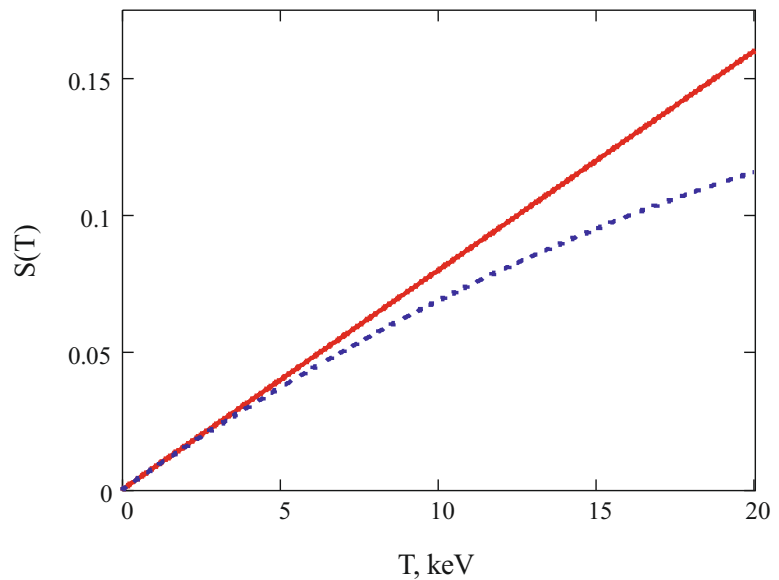

Fig. 2. The spectral slopes of the non-relativistic SZE (solid curve) and of the relativistically correct SZE spectrum (dashed curve) calculated in the range $x=3.5-4.5$ for a thermal electron population are shown as a function of the electronic temperature $T_{\mathrm{e}}$ given in $\mathrm{keV}$.

In the relativistic treatment, the $\mathrm{CMB}$ spectral distortion due to the thermal SZE is given by the expression

$\Delta I_{\text {th }}=\frac{2\left(k T_{0}\right)^{3}}{(h c)^{2}} \cdot \tau \int \mathrm{d} s P_{1}(s)\left(\frac{x^{3} \mathrm{e}^{-3 s}}{\exp \left(x \mathrm{e}^{-s}\right)-1}-\frac{x^{3}}{\mathrm{e}^{x}-1}\right)$

where

$P_{1}(s)=\int \mathrm{d} \beta f_{\mathrm{e}}(\beta) P(s, \beta)$

with $f_{\mathrm{e}}(\beta)$ being the velocity spectrum of the electron population and $P(s, \beta)$ being the probability that a single scattering of a CMB photon off an electron with speed $\beta c$ causes a logarithmic frequency shift $s \equiv \ln \left(v^{\prime} / v\right)$. Here, we use the first order approximation in $\tau$ of the photon redistribution function $P(s)$ for the sake of simplicity. Analogous conclusions hold, nonetheless, for the more general derivation (see e.g. Colafrancesco et al. 2003).

The series expansion of $i(x)$ around the crossover frequency $X_{0}$ was calculated in Eq. (A.11) of the Appendix. Using the expression for the crossover frequency $X_{0}\left(T_{\mathrm{e}}\right)=3.830(1+$ $\left.1.162 \theta_{\mathrm{e}}-0.8144 \theta_{\mathrm{e}}^{2}\right)$ given by Dolgov et al. (2001), one can calculate the slope of the thermal SZE in the relativistic treatment as a function of $k T_{\mathrm{e}}$. A good approximation of the slope $S$ in the relativistic treatment, and in the temperature range $k T_{\mathrm{e}}=1-20 \mathrm{keV}$, is given by

$$
\begin{aligned}
S_{\text {rel }} & =4.25 \frac{k T_{\mathrm{e}}}{m_{\mathrm{e}} c^{2}}-30\left(\frac{k T_{\mathrm{e}}}{m_{\mathrm{e}} c^{2}}\right)^{2} \\
& =0.08 \frac{k T_{\mathrm{e}}}{10 \mathrm{keV}}\left(1-0.138 \frac{k T_{\mathrm{e}}}{10 \mathrm{keV}}\right)
\end{aligned}
$$

and, again, depends only on the cluster temperature $T_{\mathrm{e}}$. The slopes of the non-relativistic and relativistically correct thermal SZE spectrum are shown in Fig. 2 for comparison. The value of the crossover frequency of the total SZE in the relativistic treatment is given by

$$
\begin{aligned}
X_{0, \text { total }, \text { rel }}= & X_{0}\left(T_{\mathrm{e}}\right)+\frac{V_{r}}{c} \frac{h\left[X_{0}\left(T_{\mathrm{e}}\right)\right]}{S_{\text {rel }}} \\
\approx & X_{0}\left(T_{\mathrm{e}}\right)+\frac{0.193}{1-0.138 \cdot \frac{k T_{\mathrm{e}}}{10 \mathrm{keV}}} \\
& \times\left(\frac{V_{r}}{10^{3} \mathrm{~km} \mathrm{~s}^{-1}} \cdot \frac{10 \mathrm{keV}}{k T_{\mathrm{e}}}\right)
\end{aligned}
$$




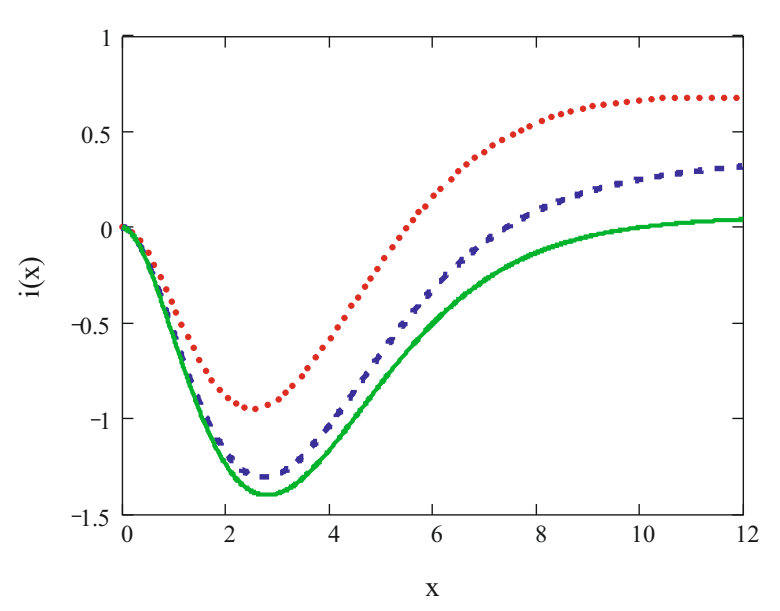

Fig. 3. The spectral shape of the normalized CMB intensity change $i(x)$ evaluated for a power-law electron spectrum with a minimum momentum $p_{1}=1$ (dotted line), 3 (dashed) and 10 (solid).

(see the Appendix for a derivation) where $X_{0}\left(T_{\mathrm{e}}\right)=3.83(1+$ $\left.1.162 \cdot \theta_{\mathrm{e}}-0.814 \cdot \theta_{\mathrm{e}}^{2}\right)$. These results show that, while the value of the crossover frequency $X_{0 \text {,total,rel }}$ depends on both $k T_{\mathrm{e}}$ and on $V_{r}$ (see Eqs. (18) and (A.13)), the value of $S$ does not depend on $V_{r}$ in the frequency range around the crossover frequency $X_{0}$ (see Appendix A for details). This is because the slope of the kinetic SZE is approximately zero in this frequency range. Therefore, a measure of $X_{0}$ for the thermal SZE cannot provide an unambiguous estimate of the cluster temperature. In contrast, a measure of the slope of the thermal SZE can provide unbiased constraints on the value of the electron temperature and, hence, the nature of the electron plasma.

In the following sections, we demonstrate that this is a general result for the SZE that can be applied to the case of other electron plasmas residing in cluster atmospheres.

\section{The spectrum of the non-thermal SZE}

We consider a non-thermal electron population with a power-law spectrum of the form

$$
\begin{aligned}
& f_{\mathrm{e}}(p)=\frac{\alpha-1}{p_{1}^{1-\alpha}-p_{2}^{1-\alpha}} \cdot p^{-\alpha} p_{1}<p<p_{2} \\
& f_{\mathrm{e}}(p)=\quad 0 \quad \text { elsewhere }
\end{aligned}
$$

where $f_{\mathrm{e}}(p)$ is the momentum distribution, and the momentum $p=\beta \gamma$ is normalized to unity. This electron spectrum is expressed as a function of $\beta$ by

$$
f_{\mathrm{e}}(\beta)=\frac{\alpha-1}{p_{1}^{1-\alpha}-p_{2}^{1-\alpha}} \cdot\left(\frac{\beta}{\sqrt{1-\beta^{2}}}\right)^{-\alpha} \cdot\left(\frac{1}{1-\beta^{2}}\right)^{3 / 2} .
$$

The spectral shape of the quantity $i(x)$ evaluated for the previous power-law electron spectrum is shown in Fig. 3 for different values of the minimum momentum $p_{1}$ of the electron spectrum with a value of the momentum $p_{2}=1000$.

The approximate value of the crossover frequency of the non-thermal SZE for a monoenergetic electron spectrum in the limit $\gamma \gg 1$ has been derived in the Appendix, and is given by

$X_{0, \text { non-th }} \approx 2 \ln \left(\frac{4}{3} \gamma^{2}\right)$.

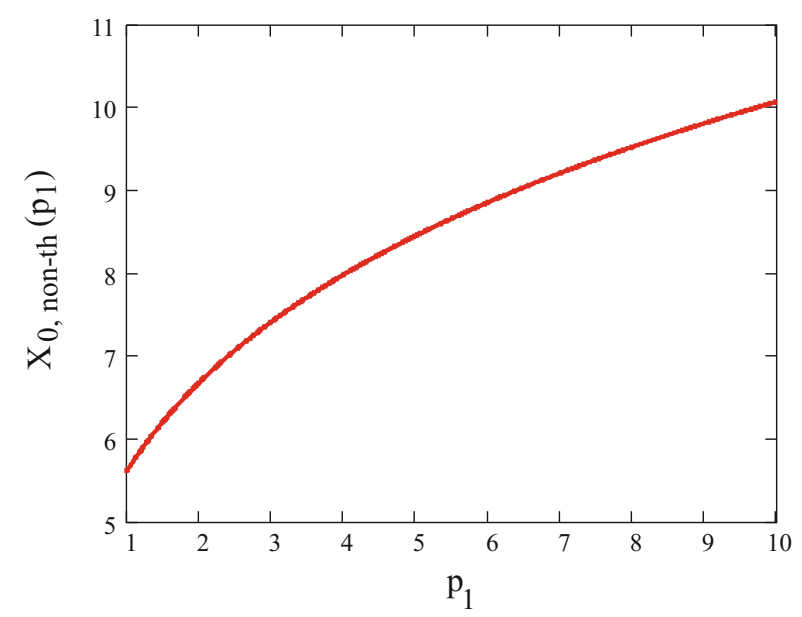

Fig. 4. The dependence of $X_{0, \text { non-th }}$ on the lower cutoff momentum $p_{1}$. We do not consider here the effect of a possible kinematic SZ effect.

The dependence of $X_{0, \text { non-th }}$ on the lower momentum $p_{1}$ of the power-law electron spectrum is shown in Fig. 4 (this figure shows the values of $X_{0, \text { non-th }}$ without the effect of the peculiar velocity).

The quantity $X_{0, \text { non-th }}$ is assumed to have values larger than $x \gtrsim 5$ for all values $p_{1} \geq 1$ (see Fig. 4). This means that for a system containing only a non-thermal electron population, the value of $X_{0, \text { non-th }}$ (and hence of the energetics/pressure of the relative electron population) cannot be determined without a precise knowledge of its peculiar velocity $V_{r}$.

The value of the slope of the non-thermal SZE is also affected by the presence of a kinematic SZE in this case. However, both the $\mathrm{SZ}_{\mathrm{kin}}$ and primary $\mathrm{CMB}$ anisotropies have a flat spectral shape in the frequency range $x=3.5-4.5$, so that their contribution to the slope of the total SZE is negligible in this frequency range.

\subsection{Combination of thermal and non-thermal SZE}

For the case of realistic clusters with radio halos and/or X-ray cavities (i.e. those clusters definitely containing a population of relativistic electrons whose energy spectrum is often represented by a power-law distribution, see Colafrancesco 2007, for a review), there is usually a cospatial distribution of thermal and non-thermal plasmas. We calculate the values of the cross-over frequency $X_{0}$ and the slope $S$ of the SZE for a combination of a thermal plus a power-law electron spectra with the addition of a peculiar velocity of the cluster.

In this case, we found that the value of the crossover frequency $X_{0}$ depends on the cluster temperature, the peculiar velocity, and the optical depths of the thermal and non-thermal electron populations

$$
\begin{aligned}
X_{0}= & 3.83+0.193\left(\frac{V_{r}}{10^{3} \mathrm{~km} \mathrm{~s}^{-1}} \frac{10 \mathrm{keV}}{k T_{\mathrm{e}}}\right) \\
& +0.148\left(\frac{\tau_{\mathrm{rel}} / \tau}{0.01} \frac{10 \mathrm{keV}}{k T_{\mathrm{e}}}\right)
\end{aligned}
$$

(see Appendix for details). 
The value of the slope of the total (thermal plus non-thermal plus kinetic) SZE is given by

$$
\begin{aligned}
S= & 0.084 \frac{k T_{\mathrm{e}}}{10 \mathrm{keV}}\left(1+7 \times 10^{-3} \frac{V_{r}}{10^{3} \mathrm{~km} \mathrm{~s}^{-1}} \frac{10 \mathrm{keV}}{k T_{\mathrm{e}}}\right. \\
& \left.+0.038 \frac{\tau_{\mathrm{rel}} / \tau}{0.01} \frac{10 \mathrm{keV}}{k T_{\mathrm{e}}}\right) .
\end{aligned}
$$

To estimate specific values of $X_{0}$ and $S$ for a representative Coma-like cluster that contains both thermal and non-thermal plasmas, we choose representative values $k T_{\mathrm{e}}=8.2 \mathrm{keV}, p_{1}=$ 10 , and $\tau_{\text {rel }} / \tau=0.01$.

For the single Maxwellian spectrum, one obtains in this case the reference values $X_{0}=3.9$ and $S=0.058$. The zero of the total SZE evaluated for a combination of thermal and non-thermal populations is $X_{0}=4.09$, and the increase in the value of $X_{0}$ is in agreement with Eq. (22). The spectral slope of the total SZE for a combination of the two electron populations is $S=0.061$, and the mild increase in $S$ in this case is also in good agreement with Eq. (23).

The contribution of the kinematic SZE to the determination of $X_{0}$ cannot be ignored. Using Eq. (22), we calculated the contributions to the value of $X_{0}$ of the terms depending on $V_{r}$ and $\tau_{\text {rel }} / \tau$. The contribution of $V_{r}$ to the value of $X_{0}$ can be neglected in contrast to the contribution of the non-thermal electron population only for values $\left|V_{r}\right|<776.8 \mathrm{~km} \mathrm{~s}^{-1}\left(\frac{\tau_{\text {rel }} / \tau}{0.01}\right)$; therefore, even for values $\left(\tau_{\text {rel }} / \tau\right) \sim 0.01$, the value of $X_{0}$ is substantially affected by possible values of $V_{r}$ in the range $\sim 800-1000 \mathrm{~km} \mathrm{~s}^{-1}$, as found in the tail of the peculiar velocity distribution (with rms value of $\approx 300 \pm 80 \mathrm{~km} \mathrm{~s}^{-1}$ ) of galaxy clusters (see Giovanelli et al. 1998).

In contrast, (from Eq. (23)), the impact of the kinematic SZE (i.e. of the cluster peculiar velocity) on the slope can be neglected in comparison with the impact of the non-thermal electron population, for values $\left|V_{r}\right|<5.4 \times 10^{3} \mathrm{~km} \mathrm{~s}^{-1}\left(\frac{\tau_{\text {rel }} / \tau}{0.01}\right)$; this means that the slope of the total SZE is almost unaffected by realistic values of the cluster peculiar velocity which are well below $5000 \mathrm{~km} \mathrm{~s}^{-1}$.

\section{A specific test case: the SZE spectrum of accelerated electrons}

To test the power of the method discussed in this paper, we apply our previous analysis to the specific case of a cluster in which the SZE signal is produced by an electron population experiencing a stochastic acceleration process from a background plasma (see Dogiel et al. 2007, for an extensive discussion of this scenario).

To describe the distribution function of the accelerated electrons, we use a kinetic equation modeling the influence of both Coulomb collision and stochastic acceleration, which is valid at sub-relativistic and relativistic energies (see e.g. Dogiel 2000; Liang et al. 2002; Dogiel et al. 2007)

$\frac{\partial f_{\mathrm{e}}}{\partial t}-\frac{1}{p^{2}} \frac{\partial}{\partial p}\left(A(p) \frac{\partial f_{\mathrm{e}}}{\partial p}+B(p) f_{\mathrm{e}}\right)=0$.

Here

$B(p)=p^{2}\left(\frac{\mathrm{d} p}{\mathrm{~d} t}\right)_{i}$

and

$A(p)=B(p) \frac{\gamma}{\left(\gamma^{2}-1\right)^{1 / 2}}\left(\frac{k T_{\mathrm{e}}}{m_{\mathrm{e}} c^{2}}\right)^{1 / 2}+p^{2} D_{\mathrm{p}}(p)$, where $D(p)=\alpha p^{q}$ is the diffusion coefficient due to the stochastic acceleration (see e.g. Dogiel et al. 2007). The rate of ionization losses is

$$
\begin{aligned}
\left(\frac{\mathrm{d} p}{\mathrm{~d} t}\right)_{i}= & \frac{1}{p} \sqrt{p^{2}+\frac{m_{\mathrm{e}} c^{2}}{k T_{\mathrm{e}}}} \frac{\gamma}{\left(\gamma^{2}-1\right)^{1 / 2}} \\
& \times\left[\ln \left(\frac{E(p) m_{\mathrm{e}} c^{2}\left(\gamma^{2}-1\right)}{h^{2} \omega_{\mathrm{p}}^{2} \gamma^{2}}\right)+0.43\right],
\end{aligned}
$$

where $\gamma=1+E(p) / m_{\mathrm{e}} c^{2}$, and $\omega_{\mathrm{p}}$ is the plasma frequency. Finally, the electron distribution function takes the form (Dogiel et al. 2007)

$$
f_{\mathrm{e}}=C \cdot \exp \left(-\int_{0}^{p} \mathrm{~d} v \frac{B(v)}{A(v)}\right) G(p)
$$

where

$$
C=\frac{\exp \left(-1 / \theta_{\mathrm{e}}\right) \sqrt{\theta_{\mathrm{e}}}}{K_{2}\left(1 / \theta_{\mathrm{e}}\right)}
$$

and

$$
G(p)=1-\frac{\int_{0}^{p} \frac{\mathrm{d} w}{A(w)} \exp \left(\int_{0}^{w} \mathrm{~d} t B(t) / A(t)\right)}{\int_{0}^{\infty} \frac{\mathrm{d} w}{A(w)} \exp \left(\int_{0}^{w} \mathrm{~d} t B(t) / A(t)\right)} .
$$

The integral

$$
\int_{0}^{p} \frac{B(v)}{A(v)} \mathrm{d} v=\int_{0}^{p} \frac{v \mathrm{~d} v}{\sqrt{1+\theta_{\mathrm{e}} v^{2}}\left(1+\alpha_{*} \theta_{\mathrm{e}} v^{q+3}\left(\frac{1}{1+\theta_{\mathrm{e}} v^{2}}\right)^{3 / 2}\right)}
$$

depends on the acceleration parameter

$\alpha_{*}=\alpha \times\left[\ln \left(\frac{E(p) m_{\mathrm{e}} c^{2}\left(\gamma^{2}-1\right)}{h^{2} \omega_{\mathrm{p}}^{2} \gamma^{2}}\right)+0.43\right]$.

The velocity distribution of the accelerated electrons is given by

$P_{\mathrm{e}}\left(\beta, \alpha_{*}\right)=\frac{\beta^{2}}{\theta_{\mathrm{e}}^{3 / 2}\left(1-\beta^{2}\right)^{5 / 2}} \cdot f\left(\frac{\beta}{\sqrt{\theta_{\mathrm{e}}\left(1-\beta^{2}\right)}}, \alpha_{*}\right)$.

The resulting spectrum of the background and accelerated particles is shown in Fig. 5. The following limiting cases hold:

i) if $\alpha_{*}=0$, the spectrum is a relativistic Maxwellian spectrum

$$
P_{\mathrm{e}}(\beta)=\frac{\gamma^{5} \beta^{2} \exp \left(-\gamma / \theta_{\mathrm{e}}\right)}{\theta_{\mathrm{e}} K_{2}\left(1 / \theta_{\mathrm{e}}\right)}
$$

where $K_{2}$ is the modified Bessel function of second kind;

ii) if $p \ll 1 / \sqrt{\theta_{\mathrm{e}}}$, the electron spectrum takes the form of the non-relativistic spectrum derived by Gurevich (1960)

$$
\begin{aligned}
f_{\mathrm{e}}(p)= & \sqrt{\frac{2}{\pi}}\left[\exp \left(-\int_{0}^{p} \frac{\mathrm{d} w}{1 / w+\alpha_{*} \theta_{\mathrm{e}} w^{q+2}}\right)\right. \\
& \left.-\exp \left(-\int_{0}^{\infty} \frac{\mathrm{d} w}{1 / w+\alpha_{*} \theta_{\mathrm{e}} w^{q+2}}\right)\right] .
\end{aligned}
$$

The position of the cut-off in the electron spectrum is, in principle, a free parameter to be fixed at any energy above the range of available X-ray observations, e.g. at $E>80 \mathrm{keV}$ (i.e. the upper value of the energy range of the Beppo-SAX PDS instrument). In our analysis, we consider specifically a value of the cut-off energy $E_{\text {cutoff }}=m_{\mathrm{e}} c^{2}(\gamma-1)=212 \mathrm{keV}$, corresponding to a value of $p_{\text {cutoff }}=1 / \sqrt{\theta_{\mathrm{e}}}$, where $\gamma=\sqrt{1+\theta_{\mathrm{e}} p^{2}}$ that is able to 


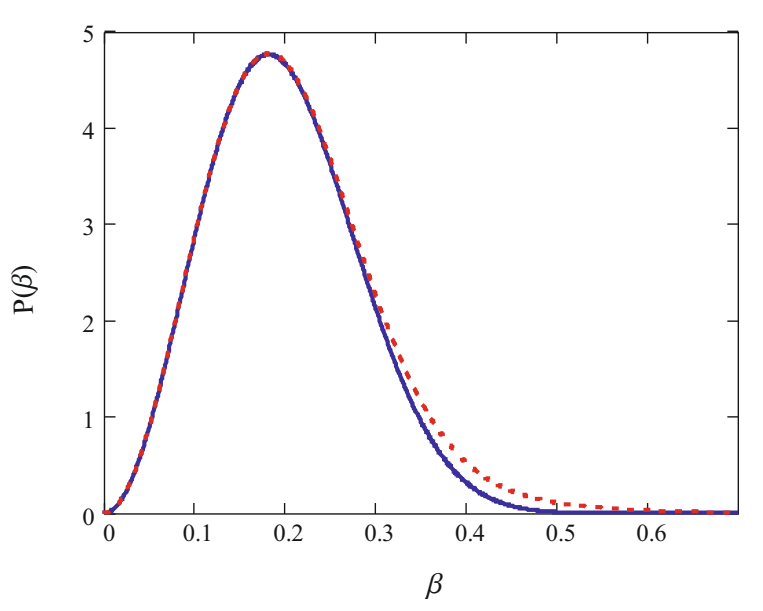

Fig. 5. The spectrum of stochastically accelerated electrons with acceleration parameter $\alpha=0.0008$ (dashed curve) is compared with the Maxwellian spectrum (solid curve). A background plasma temperature of $k T_{\mathrm{e}}=8.2 \mathrm{keV}$ is assumed here.

reproduce both the soft and hard X-ray spectrum of Coma (see Dogiel et al. 2007). In such a model for the origin of the hard $\mathrm{X}$-ray emission from the Coma cluster, we derived a value of the acceleration parameter $\alpha=0.0008$, given the value of the electron temperature of Coma $k T_{\mathrm{e}}=8.2 \mathrm{keV}$ (Dogiel et al. 2007). These parameters fix the shape of the spectrum of accelerated electrons and, therefore, the spectral shape of the relative SZE.

\subsection{The dependence of $X_{0}$ and of $S$ on the acceleration parameter}

We calculate the dependence on the value of $\alpha$ of both $X_{0}$ and $S$ for the total SZE of the considered cluster for the specific model previously discussed. For the original Maxwellian spectrum of Coma with $k T_{\mathrm{e}}=8.2 \mathrm{keV}$, we obtain values $X_{0}=3.9$ and $S=$ 0.058 in the range $x=3.5-4.5$.

We use the relativistic spectrum of thermal electron population (see Sect. 2) with values of the acceleration parameter $\alpha$ in the range $\alpha=0-0.0015$, bracketing the value $\alpha=0.0008$ obtained from the fit to the hard X-ray spectrum of Coma (Dogiel et al. 2007).

The approximated expression of the crossover frequency $X_{0}(\alpha)$ up to order $O\left(\alpha^{2}\right)$ is given by

$X_{0}\left(\alpha ; k T_{\mathrm{e}}=8.2 \mathrm{keV}\right)=3.9+15.73 \alpha+15402 \alpha^{2}$.

For the value $\alpha=0.0008$, one obtains $X_{0}=3.922$. This value of $X_{0}$ would correspond to a thermal electron spectrum (without acceleration) at an effective temperature of $k T_{\mathrm{e}}=10.5 \mathrm{keV}$.

We note that in this case the shifts induced on the value of $X_{0}$ due to both the electron acceleration process and the presence of a peculiar velocity of the cluster are intertwined. The expression for the crossover frequency, taking into account the kinematic $\mathrm{SZE}$, is given by

$X_{o, \text { total }}(\alpha)=X_{0}(\alpha)+\frac{V_{r}}{c} \frac{h\left[X_{0}(\alpha)\right]}{S(\alpha)}$.

The peculiar velocity of the Coma cluster was estimated to be $V_{r}=-29 \pm 299 \mathrm{~km} \mathrm{~s}^{-1}$ (Colless et al. 2001), therefore $X_{0, \text { total }}$ is in the range 3.84-4.00 for a value $\alpha=8 \times 10^{-4}$. Since the values $X_{0}(\alpha=0)$ and $X_{0}\left(\alpha=8 \times 10^{-4}\right)$ lie in this range, the ability to study the nature of a possible population of supra-thermal electrons directly from the displacement of the crossover frequency is quite limited.

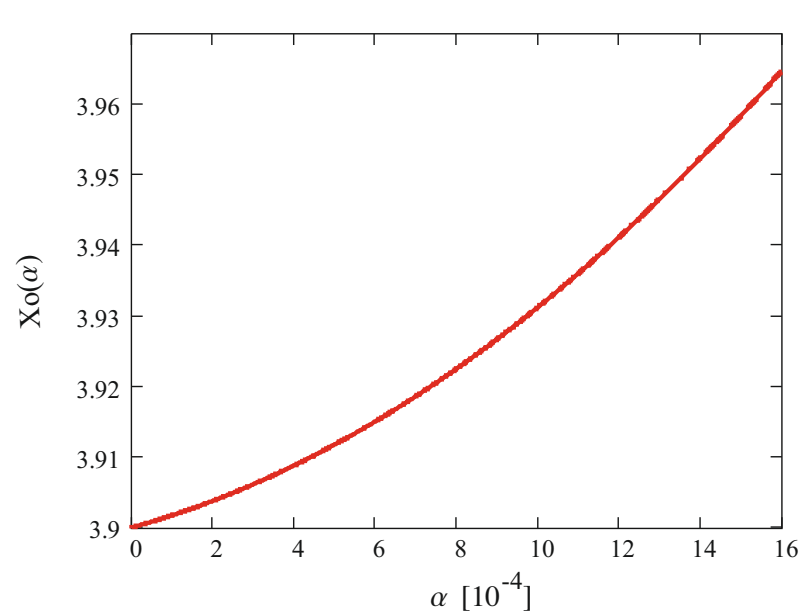

Fig. 6. The dependence of the crossover frequency $X_{0}$ on the acceleration parameter $\alpha$ given in units of $10^{-4}$.

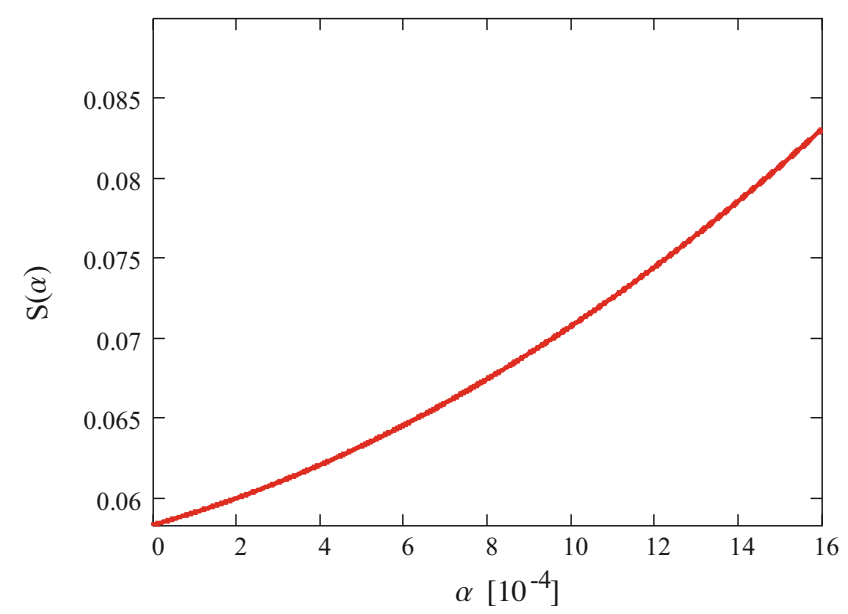

Fig. 7. The dependence of the slope $S$ of the SZE in Coma on the acceleration parameter $\alpha$ given in units of $10^{-4}$.

The value of the SZE slope (that, we recall, does not depend on the peculiar velocity in the frequency range $x=3.5-4.5)$, is therefore an important quantity for deriving useful information about the nature of supra-thermal electrons.

The approximated expression of the quantity $S(\alpha)$ up to $O\left(\alpha^{2}\right)$ is given by

$S\left(\alpha ; k T_{\mathrm{e}}=8.2 \mathrm{keV}\right)=0.0583+7.35 \cdot \alpha+5064 \cdot \alpha^{2}$

and its value is 0.0674 for an electron spectrum with $\alpha=0.0008$. The value of $S$ increases by $\approx 16 \%$ with respect to the case of the Maxwellian spectrum (i.e. with $\alpha=0$ ). This value of $S$ would correspond to a genuine thermal electron spectrum (i.e. without stochastic acceleration) with an effective temperature of $k T=9.3 \mathrm{keV}$. The dependence of the slope $S$ on the acceleration parameter is shown in Fig. 7.

\subsection{Contribution of the kinematic SZE to the slope of the total SZE}

The total SZE intensity in the case of a cluster with an in-situ accelerated electron population moving along the line of sight with a peculiar velocity $V_{r}$ is due to the combination of $S Z_{\text {th }}$ and $S Z_{\text {kin }}$

$i(x)=i_{\mathrm{th}}(x)-\frac{V_{r}}{c} h(x)$. 


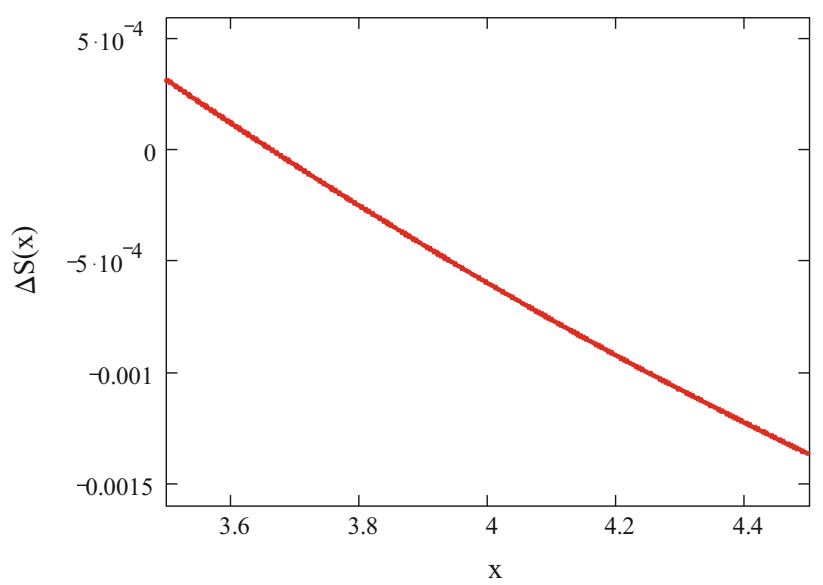

Fig. 8. The contribution of the kinematic SZE to the slope of the total SZE (see text for details).

The series expansion of the term $S Z_{\text {th }}$ around the adimensional frequency $x=4$ is given by

$i_{\text {th }}=i_{\text {th } 0}+S \cdot(x-4)+O(x-4)^{2}$,

where $S$ is the value of the slope of the SZE without the contribution of the kinematic SZ effect.

The series expansion of $h(x)$ around $x=4$ is

$h(x)=4.865-0.181(x-4)-0.512(x-4)^{2}+O(x-4)^{3}$,

which can be written as

$h(x)=h_{0}+\Delta(x) \cdot(x-4)$,

where $\Delta(x)=-0.181-0.512(x-4)+O(x-4)^{2}$.

The total SZE intensity is then given by the expression

$i(x)=i_{\text {th } 0}-\frac{V_{r}}{c} h_{0}+\left(S_{\text {th }}-\frac{V_{r}}{c} \Delta(x)\right) \cdot(x-4)$.

For a narrow frequency range, the spectral slope of the SZE can be derived by means of SZE measurements at two nearby frequencies, given by

$S_{\mathrm{obs}}=\frac{i\left(x_{1}\right)-i\left(x_{2}\right)}{x_{1}-x_{2}}$.

For the sake of clarity, we fix the first frequency $x_{1}=4$ and the second frequency lies in the range $x_{2}=[3.5,4.5]$.

In this case, we find that

$S_{\text {obs }}=S_{\text {th }}-\frac{V_{r}}{c} \Delta\left(x_{2}\right)$.

The contribution of the kinematic SZE to the slope of the total SZE is given by

$S_{\text {kin }}=S_{\text {obs }}-S_{\text {th }}=-\frac{V_{r}}{10^{3} \mathrm{~km} \mathrm{~s}^{-1}} \Delta S\left(x_{2}\right)$,

where the quantity $\Delta S\left(x_{2}\right)=3.3 \times 10^{-3} \Delta\left(x_{2}\right)$ is plotted in Fig. 8 .

It is clear that the contribution of the kinematic SZE to the slope of the total SZE is far smaller than the slope increase due to the stochastic acceleration process (see Fig. 7). It follows that the determination of the slope of the SZE is a powerful tool for studying the presence and the nature of a possible population of accelerated electrons at supra-thermal energies in the atmospheres of galaxy clusters.

\section{Discussion and conclusions}

We demonstrated in this paper that the value of the crossover frequency $X_{0}$ of the SZE depends crucially on the cluster peculiar velocity $V_{r}$ (as well as on the CMB primary anisotropies), while the value of the slope of the SZE does not depend on the kinematic SZE spectrum (and on the CMB anisotropy spectrum), in the optimal frequency range around the crossover frequency of the thermal SZE, i.e. in the frequency range $x=3.5-4.5$. This is because both the spectrum of the kinetic SZE and that of the CMB primary anisotropies are almost flat in this frequency range. Therefore, while the amplitude of the $S Z_{\text {kin }}$ produces a systematic bias in the position of the crossover frequency $X_{0}$, it does not affect significantly the slope $S$ of the SZE.

This fact is more evident when one computes the CMB temperature change produced by the SZE, i.e. the quantity

$\frac{\Delta T}{T_{0}}=\frac{\left(\mathrm{e}^{x}-1\right)^{2}}{x^{4} \mathrm{e}^{x}} \frac{\Delta I}{I_{0}}$

in fact, the CMB temperature change induced by the kinetic SZE has a flat spectrum in frequency, while any other CMB temperature change induced by other SZE have spectra with specific values of slopes that differ from zero.

Since the cluster peculiar velocity is unknown in many cases, the precise position of the crossover frequency $X_{0}$ of the thermal effect cannot be well-defined from the SZE observations because the measurements of peculiar velocity are highly uncertain.

To overcome this systematic bias, we have proposed to use the spectral slope of the SZE to obtain unbiased information about specific properties of various electron distributions in galaxy clusters: the gas temperature for a thermal electron population, the power-law spectrum of a non-thermal electron population, the combined spectrum of thermal and non-thermal electron populations, the acceleration parameter of a stochastically accelerated electron population. We have evaluated analytically specific values of the slope for various SZE signals in the frequency range $x=3.5-4.5$. We found that in this frequency range (around the crossover frequency of the thermal SZE) the spectral slope of the SZE does not depend on the value of the cluster peculiar velocity because the spectrum of the kinetic SZE is extremely flat in this frequency region. We have shown this for different electron populations residing in clusters.

A doubtless advantage of the method that we have presented here is that it provides an opportunity to search more reliably for nonthermal components of electron spectra in clusters and determine their characteristics. These allow us to derive independently parameters of acceleration processes in clusters which are usually unknown and estimated in the framework of different models. This final circumstance is particularly important for the problem of the origin of nonthermal emission from clusters in the Extreme UV, in the hard X-ray and at radio wavelengthes.

Spectroscopic measurements of the SZE spectra will become available in the near future by means of dedicated space experiments (such as e.g. SAGACE; Spectroscopic Active Galaxy And Cluster Explorer, see http://oberon.roma1.infn.it/ sagace/) based on microwave spatially resolved spectroscopic techniques. The advent of these spectroscopic capabilities will allow us to study in an unbiased way several physical details of the leptonic structure of the atmospheres of galaxy clusters and other cosmic structures.

Acknowledgements. The authors acknowledge discussions with P. De Bernardis and S. Masi. The SAGACE experiment has been selected for Phase-A study by the Italian Space Agency (ASI) as a small-mission project for the ASI space 
program 2008-2012. D.P. and V.D. are partly supported by the RFBR grant 0802-00170-a, the NSC-RFBR Joint Research Project 95WFA0700088 and by the grant of a President of the Russian Federation "Scientific School of Academician V. L. Ginzburg".

\section{Appendix A: The shape of the SZE around the crossover frequency}

In this Appendix, we calculate the values of the crossover frequency $X_{0}$ and the slope $S$ of the SZE in the frequency range $x=3.5-4.5$. We evaluate these quantities for various cases of thermal and non-thermal electron populations with the inclusion of the kinematic SZE.

\section{A.1. Thermal electron spectrum}

The total SZE intensity, in the case of a cluster with a single thermal electronic population moving along the line of sight with a peculiar velocity $V_{r}$ is due to the combination of $\mathrm{SZ}_{\text {th }}$ and $\mathrm{SZ}_{\text {kin }}$ and is given by

$i(x)=\frac{k T_{\mathrm{e}}}{m_{\mathrm{e}} c^{2}}\left[g(x)-\frac{V_{r}}{c} \frac{m_{\mathrm{e}} c^{2}}{k T_{\mathrm{e}}} h(x)\right]$.

In the non-relativistic case, the series expansion of $g(x)$ and of $h(x)$ around $x=4$ (i.e. the central value of the frequency range $x \approx 3.5-4.5$ ) is given by

$$
\begin{aligned}
g(x)= & 0.726+4.28(x-4)-0.038(x-4)^{2}-0.489(x-4)^{3} \\
& +O(x-4)^{4}
\end{aligned}
$$

and

$$
\begin{aligned}
h(x)= & 4.865-0.181(x-4)-0.512(x-4)^{2}+0.088(x-4)^{3} \\
& +O(x-4)^{4} .
\end{aligned}
$$

Thus, the series expansion of $i(x)$ around $x=4$ is given by

$$
\begin{aligned}
i(x)= & \frac{k T_{\mathrm{e}}}{m_{\mathrm{e}} c^{2}}\left[\left(0.726-4.865 \frac{V_{r}}{c} \frac{m_{\mathrm{e}} c^{2}}{k T_{\mathrm{e}}}\right)\right. \\
& \left.+\left(4.28+0.181 \frac{V_{r}}{c} \frac{m_{\mathrm{e}} c^{2}}{k T_{\mathrm{e}}}\right)(x-4)\right] \\
= & \frac{k T_{\mathrm{e}}}{m_{\mathrm{e}} c^{2}}\left[\left(0.726-0.827 \frac{V_{r}}{10^{3} \mathrm{~km} \mathrm{~s}^{-1}} \frac{10 \mathrm{keV}}{k T_{\mathrm{e}}}\right)\right. \\
& \left.+\left(4.28+0.0308 \frac{V_{r}}{10^{3} \mathrm{~km} \mathrm{~s}^{-1}} \frac{10 \mathrm{keV}}{k T_{\mathrm{e}}}\right)(x-4)\right],
\end{aligned}
$$

where we neglect higher order terms in the frequency range $x \approx 3.5-4.5$ (i.e. $v \approx 200-255 \mathrm{GHz}$ ). In the first brackets of Eq. (A.4), the term depending on $V_{r}$ and $T_{\mathrm{e}}$ cannot be neglected, but, in the second brackets of the same Eq. (A.4) we can neglect the term depending on the combination $V_{r} m_{\mathrm{e}} c^{2} / c k T_{\mathrm{e}}$, since it is always far smaller than the value 4.28 for realistic values of $V_{r}$ and $k T_{\mathrm{e}}$ found for clusters.

The slope, $S \equiv\left[i(x)-i\left(X_{0}\right)\right] /\left(x-X_{0}\right)=i(x) /\left(x-X_{0}\right)$ of the non-relativistic SZE spectrum is

$S_{\mathrm{nr}}=\frac{k T_{\mathrm{e}}}{m_{\mathrm{e}} c^{2}} \frac{g\left(X_{0}+\delta x\right)}{\delta x} \approx 4.25 \frac{k T_{\mathrm{e}}}{m_{\mathrm{e}} c^{2}}=0.08 \frac{k T_{\mathrm{e}}}{10 \mathrm{keV}}$.

Hence, the slope of the SZE intensity $i(x)$ in this frequency range does not depend on the cluster peculiar velocity.
The value of $X_{0}$ of the total (thermal plus kinematic) SZE is defined by the condition $i\left(X_{0}\right)=0$, or

$$
\frac{k T_{\mathrm{e}}}{m_{\mathrm{e}} c^{2}}\left[\left(0.726-4.865 \frac{V_{r}}{c} \frac{m_{\mathrm{e}} c^{2}}{k T_{\mathrm{e}}}\right)+4.28\left(X_{0}-4\right)\right]=0 .
$$

From the previous condition, one obtains

$X_{0, \text { total,nr }}=\left(4-\frac{0.726}{4.28}\right)+\frac{4.865}{4.28} \frac{V_{r}}{c} \frac{m_{\mathrm{e}} c^{2}}{k T_{\mathrm{e}}}$,

which is written, in more practical units, as

$X_{0, \text { total,nr }}=3.83+0.193\left(\frac{V_{r}}{10^{3} \mathrm{~km} \mathrm{~s}^{-1}} \cdot \frac{10 \mathrm{keV}}{k T_{\mathrm{e}}}\right)$.

In the relativistic treatment, the CMB spectral distortion due to the thermal SZE is given by the expression

$$
\Delta I_{\text {th }}=\frac{2\left(k T_{0}\right)^{3}}{h^{2} c^{2}} \cdot \tau \int \mathrm{d} s P_{1}(s)\left(\frac{x^{3} \mathrm{e}^{-3 s}}{\exp \left(x \mathrm{e}^{-s}\right)-1}-\frac{x^{3}}{\mathrm{e}^{x}-1}\right)
$$

where

$P_{1}(s)=\int \mathrm{d} \beta f_{\mathrm{e}}(\beta) P(s, \beta)$

with $f_{\mathrm{e}}(\beta)$ being the velocity spectrum of the electronic population and $P(s, \beta) \mathrm{d} s$ being the probability that a single scattering of a CMB photon off an electron with speed $\beta c$ causes a logarithmic frequency shift $s \equiv \ln \left(v^{\prime} / v\right)$. Here, we use the first order approximation in $\tau$ of the photon redistribution function $P(s)$ for the sake of simplicity. Analogous conclusions hold, nonetheless, for the more general derivation (see e.g. Colafrancesco et al. 2003).

The series expansion of $i(x)$ around the crossover frequency $X_{0}$ is written as

$$
\begin{aligned}
i(x)= & \int \mathrm{d} s P_{1}(s)\left[\frac{X_{0}^{3} \exp \left(X_{0} \exp (-s)\right) \exp (-4 s)}{\left(\exp \left(X_{0} \exp (-s)\right)-1\right)^{2}}\right. \\
& +\frac{X_{0}^{3} \exp \left(X_{0}\right)}{\left(\exp \left(X_{0}\right)-1\right)^{2}}+\frac{3}{X_{0}}\left(\frac{X_{0}^{3} \exp (-3 s)}{\exp \left(X_{0} \exp (-s)\right)-1}\right. \\
& \left.\left.-\frac{X_{0}^{3}}{\exp \left(X_{0}\right)-1}\right)\right]\left(x-X_{0}\right) .
\end{aligned}
$$

Using the expression $X_{0}(T)=3.830\left(1+1.162 \theta_{\mathrm{e}}-0.8144 \theta_{\mathrm{e}}^{2}\right)$ Dolgov et al. (2001), one can calculate the slope of the thermal SZE for the relativistic treatment as a function of $k T_{\mathrm{e}}$.

A good approximation of the slope for the relativistic treatment, and in the temperature range $k T_{\mathrm{e}}=1-20 \mathrm{keV}$, is given by

$$
\begin{aligned}
S_{\text {rel }} & =4.25 \frac{k T_{\mathrm{e}}}{m_{\mathrm{e}} c^{2}}-30\left(\frac{k T_{\mathrm{e}}}{m_{\mathrm{e}} c^{2}}\right)^{2} \\
& =0.08 \frac{k T_{\mathrm{e}}}{10 \mathrm{keV}}\left(1-0.138 \frac{k T_{\mathrm{e}}}{10 \mathrm{keV}}\right)
\end{aligned}
$$

The value of crossover frequency of the total SZE in the relativistic treatment $X_{0, \text { total,rel }}$ is given by

$$
\begin{aligned}
X_{0, \text { total,rel }}= & X_{0}\left(T_{\mathrm{e}}\right)+\frac{V_{r}}{c} \frac{h\left(X_{0}\left(T_{\mathrm{e}}\right)\right)}{S_{\text {rel }}} \\
\approx & X_{0}\left(T_{\mathrm{e}}\right)+\frac{0.193}{1-0.138 \cdot \frac{k T_{\mathrm{e}}}{10 \mathrm{keV}}} \\
& \times\left(\frac{V_{r}}{10^{3} \mathrm{~km} \mathrm{~s}^{-1}} \cdot \frac{10 \mathrm{keV}}{k T_{\mathrm{e}}}\right)
\end{aligned}
$$




\section{A.2. Power-law, non-thermal electron spectrum}

For a power-law, electron spectrum given by

$f_{\mathrm{e}}(p)=\frac{\alpha-1}{p_{1}^{1-\alpha}-p_{2}^{1-\alpha}} \cdot p^{-\alpha} p_{1}<p<p_{2}$

$f_{\mathrm{e}}(p)=\quad 0 \quad$ elsewhere

with $p=\beta \gamma$, the electron spectrum in terms of $\beta$ is given by

$f_{\mathrm{e}}(\beta)=\frac{\alpha-1}{p_{1}^{1-\alpha}-p_{2}^{1-\alpha}} \cdot\left(\frac{\beta}{\sqrt{1-\beta^{2}}}\right)^{-\alpha} \cdot\left(\frac{1}{1-\beta^{2}}\right)^{3 / 2}$.

If $p_{1}$ is ultra-relativistic (i.e. $p_{1} \gg 1$ ), the limiting form of $i(x)$ is given by

$i_{\text {ultra-rel }}(x) \rightarrow-\frac{x^{3}}{\mathrm{e}^{x}-1}$

One can derive analytically the approximate dependence of $X_{0}$ on $\gamma$ for a monoenergetic electron spectrum. The photon density distribution for a CMB-blackbody spectrum is

$n(x)=\frac{x^{2}}{\mathrm{e}^{x}-1}$.

An electron with Lorentz factor $\gamma$ increases the frequency $x$ of a scattered photon on average by a factor $x^{\prime} / x=4 \gamma^{2} / 3$. For relativistic electrons, the photon is therefore scattered to much higher energies. The photon spectrum after a scattering is

$$
\begin{aligned}
n^{\prime}\left(x^{\prime}\right) & =n\left[x\left(x^{\prime}\right)\right] \frac{\mathrm{d} x}{\mathrm{~d} x^{\prime}} \\
& =\left(\frac{3 x^{\prime}}{4 \gamma^{2}}\right)^{2} \frac{1}{\exp \left(3 x^{\prime} / 4 \gamma^{2}\right)-1} \cdot \frac{3}{4 \gamma^{2}} .
\end{aligned}
$$

The value of $3 x^{\prime} / 4 \gamma^{2}$ is $\ll 1$ for $\gamma \gg 1$. Since one expects in this case that $X_{0}$ is much larger than 1 because of large amounts of transferred electron momenta, we can find the value of $X_{0}$ from the condition that $\exp \left(x_{0}^{\prime}\right) \gg 1$. In this case, the value of $X_{0}$ is defined by the condition

$n^{\prime}\left(x_{0}^{\prime}\right)-n\left(x_{0}^{\prime}\right)=\frac{9}{16 \gamma^{4}} x_{0}^{\prime}-\frac{\left(x_{0}^{\prime}\right)^{2}}{\exp \left(x_{0}^{\prime}\right)}=0$

Since $X_{0} \gg 1$, the condition $X_{0} \gg \ln \left(X_{0}\right)$ holds, and one obtains

$X_{0} \approx 2 \ln \left(\frac{4}{3} \gamma^{2}\right)$.

\section{A.3. Combination of thermal and non-thermal electron populations}

We evaluate the values of $X_{0}$ and $S$ for a combination of a thermal plus a power-law electron spectra with the addition of a peculiar velocity of the cluster.

One can consider a photon to be removed effectively from the $\mathrm{CMB}$ spectrum if its energy is increased by about one order of magnitude or more, requiring $\gamma>3$ (e.g. Ensslin $\&$ Kaiser 2000). Using the usual Kompaneets approximation and neglecting those relativistic electrons with $\gamma<3$, the produced distorted spectrum is

$i(x)=\frac{k T_{\mathrm{e}}}{m_{\mathrm{e}} c^{2}}\left[g(x)-\frac{V_{r}}{c} \frac{m_{\mathrm{e}} c^{2}}{k T_{\mathrm{e}}} h(x)-\frac{\tau_{\mathrm{rel}}}{\tau} \frac{m_{\mathrm{e}} c^{2}}{k T_{\mathrm{e}}} \frac{x^{3}}{\mathrm{e}^{x}-1}\right]$.

The series expansion of $i(x)$ around $x=4$ is then

$$
\begin{aligned}
i(x)= & \frac{k T_{\mathrm{e}}}{m_{\mathrm{e}} c^{2}}\left[\left(0.726-4.865 \frac{V_{r}}{c} \frac{m_{\mathrm{e}} c^{2}}{k T_{\mathrm{e}}}-1.194 \frac{\tau_{\mathrm{rel}}}{\tau} \frac{m_{\mathrm{e}} c^{2}}{k T_{\mathrm{e}}}\right)\right. \\
& \left.+\left(4.28+0.181 \frac{V_{r}}{c} \frac{m_{\mathrm{e}} c^{2}}{k T_{\mathrm{e}}}+0.321 \frac{\tau_{\mathrm{rel}}}{\tau} \frac{m_{\mathrm{e}} c^{2}}{k T_{\mathrm{e}}}\right)(x-4)\right] .
\end{aligned}
$$

From the condition $i(x)=0$, the value of the crossover frequency $X_{0}$ of the total SZE is

$$
X_{0}=4-\frac{1}{4.28} \times \frac{0.726-4.865 \frac{V_{r}}{c} \frac{m_{\mathrm{e}} c^{2}}{k T_{\mathrm{e}}}-1.194 \frac{\tau_{\mathrm{rel}}}{\tau} \frac{m_{\mathrm{e}} c^{2}}{k T_{\mathrm{e}}}}{1+\frac{0.181}{4.28} \frac{V_{r}}{c} \frac{m_{\mathrm{e}} c^{2}}{k T_{\mathrm{e}}}+\frac{0.321}{4.28} \frac{\tau_{\mathrm{rel}}}{\tau} \frac{m_{\mathrm{e}} c^{2}}{k T_{\mathrm{e}}}}
$$

From Eqs. (A.22) and (A.23), we then derive the values of

$$
\begin{aligned}
X_{0} & =4-\frac{0.726}{4.28}+\frac{4.865}{4.28} \frac{V_{r}}{c} \frac{m_{\mathrm{e}} c^{2}}{k T_{\mathrm{e}}}+\frac{1.194}{4.28} \frac{\tau_{\mathrm{rel}}}{\tau} \frac{m_{\mathrm{e}} c^{2}}{k T_{\mathrm{e}}} \\
& =3.83+0.193\left(\frac{V_{r}}{10^{3} \mathrm{~km} \mathrm{~s}^{-1}} \frac{10 \mathrm{keV}}{k T_{\mathrm{e}}}\right) \\
& +0.148\left(\frac{\tau_{\mathrm{rel}} / \tau}{0.01} \frac{10 \mathrm{keV}}{k T_{\mathrm{e}}}\right)
\end{aligned}
$$

and of the slope of the total SZE

$$
\begin{aligned}
S & =4.28 \frac{k T_{\mathrm{e}}}{m_{\mathrm{e}} c^{2}}\left(1+7 \times 10^{-3} \frac{V_{r}}{10^{3} \mathrm{~km} \mathrm{~s}^{-1}} \frac{10 \mathrm{keV}}{k T_{\mathrm{e}}}\right. \\
& \left.+0.038 \frac{\tau_{\mathrm{rel}} / \tau}{0.01} \frac{10 \mathrm{keV}}{k T_{\mathrm{e}}}\right) .
\end{aligned}
$$

\section{References}

Birkinshaw, M. 1999, Phys. Rep., 310, 97 Colafrancesco, S. 2004, A\&A, 422, L23 Colafrancesco, S. 2005, A\&A, 435, L9

Colafrancesco, S. 2007, New Astron. Rev., 51, 394 Colafrancesco, S., Marchegiani, P., \& Palladino, E. 2003, A\&A, 397, 27 Colless, M., Saglia, R. P., Burstein, D., et al. 2001, MNRAS, 321, 277 Dogiel, V. A. 2000, A\&A, 357, 66

Dogiel, V., Colafrancesco, S., Ko, C. M., et al. 2007, A\&A, 461, 433

Dolgov, A. D., Hansen, S. H., Pastor, S., \& Semikoz, D. V. 2001, ApJ, 554, 74 Ensslin, T., \& Kaiser, C. 2000, A\&A, 360, 417

Giovanelli, R., Hayness, M. P., Salzer, J. J., et al. 1998, AJ, 116, 2632

Gurevich, A. V. 1960, Sov. Phys. JETP, 38, 1150 Itoh, N., Kohyama, Y., \& Nozawa, S. 1998, ApJ, 502, 7

Liang, H., Dogiel, V. A., \& Birkinshaw, M. 2002, MNRAS, 337, 567

Kompaneets, A. S. 1957, Soviet Phys. JETP, 4, 730

Sunyaev, R. A., \& Zel'dovich, Ya. B. 1972, Comm. Astrophys. Space Sci., 4, 173

Sunyaev, R. A., \& Zel'dovich, Ya. B. 1980, ARA\&A, 18, 537 